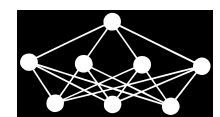

\title{
NO ROUNDING REVERSE FUZZY MORPHOLOGICAL ASSOCIATIVE MEMORIES
}

\author{
N. Feng, Y. Yao
}

\begin{abstract}
The fuzzy morphological associative memories (FMAM) have many attractive advantages, but their recall effects for hetero associative memories are poor. This shortcoming impedes the application of hetero-FMAM. Aiming at the problem, and inspired by the unified framework of morphological associative memories, a new method called no rounding reverse fuzzy morphological associative memories ( $\left.\mathrm{NR}^{2} \mathrm{FMAM}\right)$ is presented by the paper. The value of the new method lies in hetero associative memories. Analyses and experiments show that, it has significantly affected hetero associative morphological memories and with a certain noise robustness. In some cases, it can work more effectively with greater correct recall rate than FMAM. The paper analyzes the reason that $\mathrm{NR}^{2} \mathrm{FMAM}$ is sometimes better than FMAM, and thinks that no rounding neural computing is one of passable reasons. At the same time, the condition that the recall rate of $\mathrm{NR}^{2} \mathrm{FMAM}$ is greater than FMAM is given by the corresponding theorem in this paper. The $\mathrm{NR}^{2} \mathrm{FMAM}$ not only enriched the theory of the morphological associative mnemonic framework, but also helps contribute to the solution of the hetero associative mnemonic problem which is incomplete. At the same time, it can serve as a new identification technology in social networks.
\end{abstract}

Key words: fuzzy morphological associative memories, framework, hetero association, incomplete recall, no rounding

Received: April 9, 2016

DOI: $10.14311 /$ NNW.2016.26.033

Revised and accepted: October 3, 2016

\section{Introduction}

Associative memories (AM) are one of the functions of the human brain, also the source of thinking and innovation. Using computers to realize the simulations for associative memories is one of our pursuits of goals. In 1982, Hopfield proposed the famous Hopfield neural network [9], and opened a new era of simulating associative memories. However, the Hopfield network requires orthogonal input patterns.

\footnotetext{
* Naiqin Feng, Institute of Machine Learning and Big Data, School of Information Engineering, Zhengzhou University of Industrial Technology, Zhengzhou 451150, P.R. China, E-mail: fengnaiqin@163.com

†Yingle Yao - Corresponding author, School of Information Engineering, Zhengzhou University of Industrial Technology, Zhengzhou 451150, P.R. China, E-mail: fengnaiqin@163.com
} 
Meanwhile, the convergence problem with the network exists. Besides,its storage capacity is limited, no more than $15 \%$ of the total network neurons [8]. This means that a large number of neurons in the network are wasted!

Hopfield network, radial basis function network, probabilistic neural network $[10,12]$, and so on are all belonged to traditional artificial neural networks. Although they have many applications in pattern recognition and picture processing [11,13,25], classification [14], prediction, etc., their limitations are also obvious, for example, the "black box", longer training time, and poorer generalization ability.

Morphological neural networks (MNN) turned over a new leaf for the development of artificial associative memories. In 1998, Ritter et al. realized morphological associative memories (MAM) by using morphological neural networks [17]. After that, people presented some of the other theories and methods of morphological associative memories, for example, morphological bidirectional associative memories (MBAM) [16], complex morphological associative memories (CMAM) [1], and so on. Wang et al. treated input vectors and output vectors as fuzzy sets, therefore they presented the fuzzy morphological associative memories (FMAM) [24], enhanced FMAM (EFMAM) based on empirical kernel map [22] and economized EFMAM (E $E^{2}$ FMAM) [23]. Feng et al. proposed the unified framework of morphological associative memories in complex domain $\left(\mathrm{UFMAM}_{\mathrm{CD}}\right)$ [3], and the MAM based on four-dimensional storage (MAM-FDS) [7], and the Logarithmic and exponential MAM (LEMAM) [6].

Morphological associative memories have many advantages. In contrast to traditional associative memories, morphological associative memories converge in one step. Thus, convergence problems do not exist. Morphological analogues to the Hopfield network not only proved to be far more robust in the presence of noise but have also unlimited storage capacity for perfect inputs. Wang et al. also pointed out that morphological auto-associative memories (auto-MAM) and fuzzy morphological auto-associative memories (auto-FMAM) have many attractive advantages such as unlimited storage capacity, one-shot recall speed and good noise-tolerance to single erosive or dilative noise. Morphological associative memories have many applications in pattern recognition [19,21], image processing [2,5], classification and prediction [20], psychology research [4], and so on.

However, the researches and applications of morphological associative memories mainly concentrated on the auto-associative memories, but the studies and applications for hetero-associative memories were relatively less. The main reason is that hetero-associative morphological memories (HAMM) are incomplete, namely, they do not guarantee perfect recall memories, even if their inputs are complete. We call this problem "imperfectness of HAMM". This problem does not exist for auto-associative morphological memories (AAMM). In the case of complete inputs, auto-associative morphological memories will ensure complete recall memories. Although the HAMM based on four-dimensional storage can guarantee perfect recall memories for hetero-associative morphological memories in the case of complete inputs, this method obviously increases some overhead of time and space. Under the circumstances, it is not suitable for real-time processing and large-scale problems. Therefore, it is necessary to develop some different methods of MAM in order to improve the performance of HAMM. 
In this paper, aiming at the shortcoming of HAMM, we put forward a new method of MAM, called no rounding reverse FMAM (NR $\left.{ }^{2} F M A M\right)$. It is formed on the basis of UFMAM ${ }_{\mathrm{CD}}$. Firstly, it was inspired by UFMAM $\mathrm{CD}_{\mathrm{CD}}$ and secondly described by the symbol system of UFMAM $\mathrm{CD}$. It should be pointed out that $\mathrm{NR}^{2}$ FMAM and FMAM is different. FMAM is a kind of morphological associative memories "from division to multiplication", while $\mathrm{NR}^{2} \mathrm{FMAM}$ a kind of morphological associative memories "from multiplication to division". This method of NR $^{2}$ FMAM looks simple, but sometimes will bring amazing changes. Experiments show that NR ${ }^{2}$ FMAM is a very useful morphological associative mnemonic method. In many cases, it can get better effects of HAMM than FMAM. At the same time, our analysis shows that it can serve as a new identification technology in social networks.

The rest of this paper is organized as follows. A brief introduction to UFMAM $\mathrm{CD}_{\mathrm{CD}}$ is given in Section 2. Our NR ${ }^{2}$ FMAM is detailed in Section 3. Section 4 is the performance of $\mathrm{NR}^{2} \mathrm{FMAM}$ and its comparison with FMAM. This part presents the experimental results of our method. Experiments show that, in some cases, the $\mathrm{NR}^{2}$ FMAM achieved better effects on hetero-associative memories. Sometimes, its correct recall rate even approached $100 \%$ ! Why is this? Why can a simple change bring us the good results? And under what conditions, NR ${ }^{2}$ FMAM can obtain better results of hetero-associative memories than FMAM? These problems are discussed and analyzed in Section 5. The conclusions are given in Section 6.

\section{UFMAM $_{\mathrm{CD}}$}

It can bring us some inspirations to know $\mathrm{UFMAM}_{\mathrm{CD}}$ from the following five aspects.

\subsection{Computational basis of UFMAM $_{\mathrm{CD}}$}

The basic computation occurring in $\mathrm{UFMAM}_{\mathrm{CD}}$ is based on the algebraic lattice structure $(\mathbb{U}, \wedge, \vee, \bigcirc)$, where the symbol $\mathbb{U}$ denotes a set or domain, such as $\mathbb{U}=$ $\mathbb{R}(\mathbb{R}=(-\infty,+\infty)), \mathbb{U}=\mathbb{R}_{+}\left(\mathbb{R}_{+}=(0,+\infty)\right)$, or $\mathbb{U}=\mathbb{C}(\mathbb{C}=\{c \mid c=a \pm b i, a$ and $b \in \mathbb{R}, i=\sqrt{-1}\})$; the symbols $\wedge$ and $\vee$ denote the binary operations of minimum and maximum, respectively. The symbol $\bigcirc$ represents the closed operation on $\mathbb{U}$. For example, $\bigcirc$ can be arithmetical operator + or - , which is closed operation on $\mathbb{R}$. Also, it can be $\cdot$ (multiplication) or / (division), which is closed operation on $\mathbb{R}_{+}$. $\bigcirc$ can even be beyond operator, such as the logarithmic operator or exponential operator, which is closed operation on $\mathbb{R}_{+}$if we constrain that logarithmic base and logarithmic antilogarithm are all greater than 1 . We also use the symbol $\ominus$ to denote the inverse operation of $\bigcirc$. Of course, $\bigcirc$ is also the inverse operation of $\ominus$.

\subsection{Conditions accepted by UFMAM $_{\mathrm{CD}}$}

An object in UFMAM $\mathrm{CD}_{\mathrm{CD}}$ satisfies the following conditions:

(1) Ordered: if $a, b \in \mathbb{U}$, then $a \leqslant b$ or $b \leqslant a$;

(2) Closed: if $a, b \in \mathbb{U}$ and $a \bigcirc b=r$, then $r \in \mathbb{U}$; 
(3) Morphological: adopting morphological paradigm and operators in UFMAM ${ }_{\mathrm{CD}}$;

(4) Valid: following the right algorithms.

\subsection{Reciprocal inverse operations in UFMAM $\mathrm{UD}_{\mathrm{CD}}$}

UFMAM $_{\mathrm{CD}}$ pointed out that in morphological associative memories, there are two kinds of reciprocal inverse operations, namely,

(1) Reciprocal inverse operations $\wedge$ and $\vee$. They are the minimum operator and the maximum operator, respectively, and also the basic erosion and dilation operations in mathematical morphology, respectively.

(2) Reciprocal inverse operators $\bigcirc$ and $\ominus$. They are two abstract reciprocal inverse operators. To be specific, they can be + and - , also can be - and /. Of course, if the conditions accepted by UFMAM $_{\mathrm{CD}}$ are satisfied, we do not rule out the possibility that other reciprocal inverse operators are used. UFMAM $\mathrm{CD}_{\mathrm{D}}$ pointed out that among various kinds of morphological associative memories, an obvious common characteristic is that if an operator and an operation are used in the process of memories, then the corresponding inverse operator and inverse operation are must be used in the process of associative recall. In the memory phase of FMAM, for example, the memory matrix $\mathbf{A}_{\mathbf{X Y}}$ for the input pattern matrix $\mathbf{X}$ and the output pattern matrix $\mathbf{Y}$ is computed by using operator / and operation $\wedge$; or the memory matrix $\mathbf{B}_{\mathbf{X Y}} \mathbf{Y}$ for $\mathbf{X}$ and $\mathbf{Y}$ is computed by using operator / and operation $\vee$. But in the recall phase of FMAM, the $\mathbf{Y}$ is computed by using operator · and operation $\vee$ for $\mathbf{A}_{\mathbf{X Y}}$ and $\mathbf{X}$; or the $\mathbf{Y}$ is computed by using operator - and operation $\wedge$ for $\mathbf{B}_{\mathbf{X Y}}$ and $\mathbf{X}$.

\subsection{Symbols in UFMAM ${ }_{\mathrm{CD}}$}

In $\mathrm{UFMAM}_{\mathrm{CD}}$, the abstract operator $\bigcirc$ or $\ominus$, and the operation $\wedge$ or $\vee$ fuse together and transform into an organic whole, thus form the abstract morphological memory operator $\stackrel{\wedge}{\wedge}$ or $\stackrel{\ominus}{\vee}$, and the corresponding abstract morphological recall operator $\stackrel{\ominus}{\vee}$ or $\stackrel{\ominus}{\wedge}$. For arithmetic operations, we can construct eight specific morphological operators. They are $\stackrel{+}{\wedge}, \bar{\wedge}, \dot{\wedge}, \hat{\wedge}, \stackrel{+}{v}, \bar{v}, \dot{v}$ and $\dot{v}$, respectively.

\subsection{Memory and recall in $\mathrm{UFMAM}_{\mathrm{CD}}$}

Let $\left(\mathbf{x}^{1}, \mathbf{y}^{1}\right), \ldots,\left(\mathbf{x}^{k}, \mathbf{y}^{k}\right)$ be $k$ vector pairs $\operatorname{with}\left(x_{1}^{\xi}, \ldots, x_{n}^{\xi}\right) \in \mathbb{R}^{n}$ and $\left(y_{1}^{\xi}, \ldots, y_{n}^{\xi}\right) \in$ $\mathbb{R}^{m}$ for $\xi=1, \ldots, k$. For a given set of pattern associations $\left(\mathbf{x}^{\xi}, \mathbf{y}^{\xi}\right): \xi=1, \ldots, k$ we define a pair of associated pattern matrices $(\mathbf{X}, \mathbf{Y})$, where $\mathbf{X}=\left(\mathbf{x}^{1}, \ldots, \mathbf{x}^{k}\right)$ and $\mathbf{Y}=\left(\mathbf{y}^{1}, \ldots, \mathbf{y}^{k}\right)$. Thus, $\mathbf{X}$ is of dimension $n \times k$ and $\mathbf{Y}$ is of dimension $m \times k$. With each pair of matrices $(\mathbf{X}, \mathbf{Y})$ we define two natural morphological $m \times n$ memories $\mathbf{W}_{\mathbf{X Y}}$ and $\mathbf{M}_{\mathbf{X Y}}$ as follows.

Definition 1. Morphological ${ }_{\wedge}^{\wedge}$-memory $\mathbf{W}_{\mathbf{X Y}}$ is defined by

$$
\mathbf{W}_{\mathbf{X Y}}=\mathbf{Y}_{\wedge}^{\circ} \mathbf{X}^{\prime}=\bigwedge_{\xi=1}^{k}\left[\mathbf{y}^{\xi} \wedge\left(\mathbf{x}^{\xi}\right)^{\prime}\right]=\bigwedge_{\xi=1}^{k}\left[\begin{array}{llr}
y_{1}^{\xi} \bigcirc x_{1}^{\xi} & \ldots & y_{1}^{\xi} \bigcirc x_{n}^{\xi} \\
\vdots & \ddots & \vdots \\
y_{m}^{\xi} \bigcirc x_{1}^{\xi} & \ldots & y_{m}^{\xi} \bigcirc x_{n}^{\xi}
\end{array}\right] .
$$


Feng N., Yao Y.: No rounding reverse fuzzy morphological...

Its arbitrary element $w_{i j}$ is given by

$$
w_{i j}=\bigwedge_{\xi=1}^{k}\left(y_{i}^{\xi} \bigcirc x_{j}^{\xi}\right) .
$$

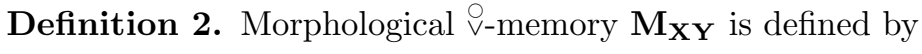

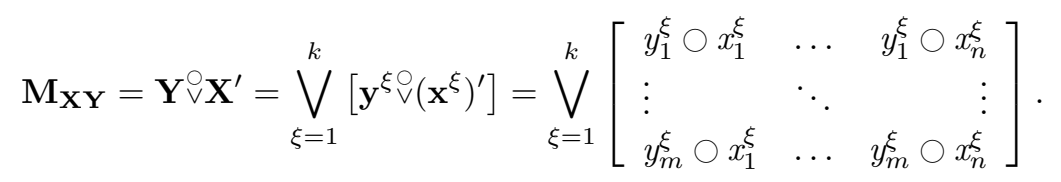

Its arbitrary element $m_{i j}$ is given by

$$
m_{i j}=\bigvee_{\xi=1}^{k}\left(y_{i}^{\xi} \bigcirc x_{j}^{\xi}\right) .
$$

Obviously, when $k=1$ and $(\mathbf{X}, \mathbf{Y})=\left(\mathbf{x}^{\xi}, \mathbf{y}^{\xi}\right)$, we have

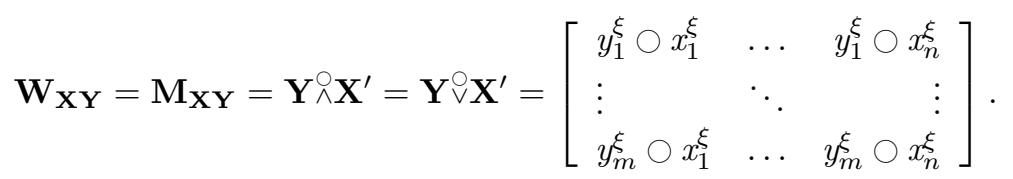

Under the stimulus of input pattern $\mathbf{x}^{\gamma}$, the morphological associative memory network generates association and recall. UFMAM $\mathrm{UD}_{\mathrm{CD}}$ also defines two abstract morphology recall paradigms with the recall pattern $\mathbf{y}$.

Definition 3. Morphological $\stackrel{\ominus}{\vee}$-recall paradigm is defined by

$$
\mathbf{y}=\mathbf{W}_{\mathbf{X Y}} \stackrel{\ominus}{\vee} \mathbf{x}^{\gamma}=\left[\begin{array}{ccc}
\bigvee_{i=1}^{n}\left(w_{1 i}\right. & \ominus & \left.x_{i}^{\gamma}\right) \\
\vdots & & \\
\bigvee_{i=1}^{n}\left(w_{m i}\right. & \ominus & \left.x_{i}^{\gamma}\right)
\end{array}\right]
$$

Definition 4. Morphological $\stackrel{\ominus}{\wedge}$-recall paradigm is defined by

$$
\mathbf{y}=\mathbf{M}_{\mathbf{X Y}} \stackrel{\ominus}{\wedge} \mathbf{x}^{\gamma}=\left[\begin{array}{ccc}
\bigwedge_{i=1}^{n}\left(m_{1 i}\right. & \ominus & \left.x_{i}^{\gamma}\right) \\
\vdots & & \\
\bigwedge_{i=1}^{n}\left(m_{m i}\right. & \ominus & \left.x_{i}^{\gamma}\right)
\end{array}\right]
$$

Distinctly, if $(\mathbf{X}, \mathbf{Y})=\left(\mathbf{x}^{\xi}, \mathbf{y}^{\xi}\right)$, namely $(\mathbf{X}, \mathbf{Y})$ has only a pair of vectors, then

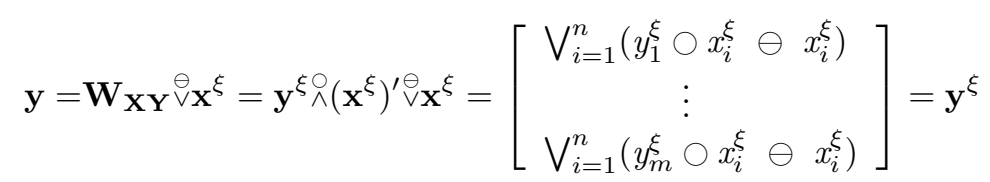

or

$$
\mathbf{y}=\mathbf{M}_{\mathbf{X Y}} \stackrel{\ominus}{\wedge} \mathbf{x}^{\xi}=\mathbf{y}^{\xi} \vee\left(\mathbf{x}^{\xi}\right)^{\prime} \wedge \mathbf{x}^{\xi}=\left[\begin{array}{c}
\bigwedge_{i=1}^{n}\left(y_{1}^{\xi} \bigcirc x_{i}^{\xi} \ominus x_{i}^{\xi}\right) \\
\vdots \\
\bigwedge_{i=1}^{n}\left(y_{m}^{\xi} \bigcirc x_{i}^{\xi} \ominus x_{i}^{\xi}\right)
\end{array}\right]=\mathbf{y}^{\xi} .
$$




\section{No rounding reverse FMAM}

We now introduce our no rounding reverse FMAM (NR ${ }^{2}$ FMAM).

\subsection{Computational basis of $\mathrm{NR}^{2}$ FMAM}

In the unified framework of morphological associative memories, for the abstract algebraic lattice structure $(\mathbb{U}, \wedge, \vee, \bigcirc)$, if we take $\mathbb{U}=\mathbb{R}_{+}, \bigcirc=\cdot$ (accordingly, $\ominus=/$ ), then the computational basis of $\mathrm{NR}^{2} \mathrm{FMAM}$ is just established.

\subsection{Memory of NR NRMAM $^{2}$ FMA}

Definition 5. In $\mathrm{NR}^{2} \mathrm{FMAM}$, the $\wedge$ Memory denoted by $\mathbf{C}_{\mathbf{X Y}}$ is defined by

$$
\mathbf{C}_{\mathbf{X Y}}=\mathbf{Y} \wedge \mathbf{X}^{\prime}=\bigwedge_{\xi=1}^{k}\left[\mathbf{y}^{\xi} \wedge\left(\mathbf{x}^{\xi}\right)^{\prime}\right]
$$

Its arbitrary element $c_{i j}$ is given by

$$
c_{i j}=\bigwedge_{\xi=1}^{k}\left(y_{i}^{\xi} \cdot x_{j}^{\xi}\right) .
$$

Definition 6. In $\mathrm{NR}^{2} \mathrm{FMAM}$, the $\dot{V}_{-}$Memory denoted by $\mathbf{D}_{\mathbf{X Y}}$ is defined by

$$
\mathbf{D}_{\mathbf{X Y}}=\mathbf{Y} \dot{\vee} \mathbf{X}^{\prime}=\bigvee_{\xi=1}^{k}\left[\mathbf{y}^{\xi} \dot{\vee}\left(\mathbf{x}^{\xi}\right)^{\prime}\right]
$$

Its arbitrary element $d_{i j}$ is given by

$$
d_{i j}=\bigvee_{\xi=1}^{k}\left(y_{i}^{\xi} \cdot x_{\mathrm{j}}^{\xi}\right) .
$$

\subsection{Recall of NR ${ }^{2} \mathrm{FMAM}$}

$\mathrm{NR}^{2} \mathrm{FMAM}$ takes into the association and recall process with the stimulation of input pattern $\mathbf{x}^{\gamma}$. The recall or output pattern can be obtained by replacing $\mathbf{W}_{\mathbf{X Y}}$ with $\mathbf{C}_{\mathbf{X Y}}$ and replacing $\ominus$ with / in the formula (6), or by replacing $\mathbf{M}_{\mathbf{X Y}}$ with $\mathbf{D}_{\mathbf{X Y}}$ and replacing $\ominus$ with / in the formula (7). The two types of recall are called '́-recall and $\wedge$-recall by us, respectively. Their definitions are as follows.

Definition 7. In $\mathrm{NR}^{2} \mathrm{FMAM}$, the ${ }^{\prime}$-recall is defined by

$$
\mathbf{C}_{\mathbf{X Y}} \vee \mathbf{X}^{\gamma}=\left[\begin{array}{c}
\bigvee_{i=1}^{n}\left(c_{1 i} / x_{i}^{\gamma}\right) \\
\vdots \\
\bigvee_{i=1}^{n}\left(c_{m i} / x_{i}^{\gamma}\right)
\end{array}\right]
$$


Feng N., Yao Y.: No rounding reverse fuzzy morphological...

Definition 8. In $\mathrm{NR}^{2} \mathrm{FMAM}$, the ${ }^{\wedge}$-recall is defined by

$$
\mathbf{D}_{\mathbf{X Y}} \wedge^{\wedge} \mathbf{x}^{\gamma}=\left[\begin{array}{c}
\bigwedge_{i=1}^{n}\left(d_{1 i} / x_{i}^{\gamma}\right) \\
\vdots \\
\bigwedge_{i=1}^{n}\left(d_{m i} / x_{i}^{\gamma}\right)
\end{array}\right]
$$

\section{Performance of $\mathrm{NR}^{2} \mathrm{FMAM}$ and its comparison with FMAM}

Theorem 1. $\mathbf{C}_{\mathbf{X Y}}$ is a $\wedge$-perfect memory for $(\mathbf{X}, \mathbf{Y})$, if and only if for each $\xi=$ $1, \ldots, k$, each row of the matrix $\left[\mathbf{y}^{\xi} \wedge\left(\mathbf{x}^{\xi}\right)^{\prime}\right]-\mathbf{C}_{\mathbf{X Y}}$ contains a zero entry.

Proof. $\mathbf{C}_{\mathbf{X Y}}$ is a $\dot{\wedge}$-perfect memory for $(\mathbf{X}, \mathbf{Y}), \forall \xi=1, \ldots, k$ and $\forall i=1, \ldots, m$

$$
\begin{aligned}
\Leftrightarrow\left(\mathbf{C}_{\mathbf{X Y}}{ }^{\prime} \mathbf{X}^{\xi}\right)_{i} & =y_{i}^{\xi} \Leftrightarrow y_{i}^{\xi}-\left(\mathbf{C}_{\mathbf{X Y}} \vee^{\prime} \mathbf{X}^{\xi}\right)_{i}=0 \\
\Leftrightarrow y_{i}^{\xi}-\bigvee_{j=1}^{n}\left(c_{i j} / x_{j}^{\xi}\right) & =0 \Leftrightarrow \bigwedge_{j=1}^{n}\left[y_{i}^{\xi}-\left(c_{i j} / x_{j}^{\xi}\right)\right]=0 \\
\Leftrightarrow \bigwedge_{j=1}^{n}\left(y_{i}^{\xi} \cdot x_{j}^{\xi}-c_{i j}\right) & =0 \Leftrightarrow \bigwedge_{j=1}^{n}\left[\left(\mathbf{y}^{\xi} \dot{\wedge}\left(\mathbf{x}^{\xi}\right)^{\prime}\right)-\mathbf{C}_{\mathbf{X Y}}\right]_{i j}=0 .
\end{aligned}
$$

This last set of equations is true if and only if for each $\xi=1, \ldots, k$, and each integer $i=1, \ldots, m$, each column entry of the $i_{t h}$ row of the matrix $\left[\mathbf{y}^{\xi} \wedge\left(\mathbf{x}^{\xi}\right)^{\prime}\right]-\mathbf{C}_{\mathbf{X Y}}$ contains at least one zero entry.

Theorem 2. $\mathbf{D}_{\mathbf{X Y}}$ is a $\dot{\mathbf{V}}$-perfect memory for $(\mathbf{X}, \mathbf{Y})$, if and only if for each $\xi=1, \ldots, k$, each row of the matrix $\mathbf{D}_{\mathbf{X Y}}-\left[\mathbf{y}^{\xi} \dot{\bigvee}\left(\mathbf{x}^{\xi}\right)^{\prime}\right]$ contains a zero entry.

Proof. $\mathbf{D}_{\mathbf{X Y}}$ is a $\dot{\bigvee}$-perfect memory for $(\mathbf{X}, \mathbf{Y}), \forall \xi=1, \ldots, k$ and $\forall i=1, \ldots, m$

$$
\begin{aligned}
\Leftrightarrow\left(\mathbf{D}_{\mathbf{X Y}} \wedge \mathbf{X}^{\xi}\right)_{i} & =y_{i}^{\xi} \Leftrightarrow\left(\mathbf{D}_{\mathbf{X Y}} \wedge \mathbf{X}^{\xi}\right)_{i}-y_{i}^{\xi}=0 \\
\Leftrightarrow \bigwedge_{j=1}^{n}\left(d_{i j} / x_{j}^{\xi}\right)-y_{i}^{\xi} & =0 \Leftrightarrow \bigwedge_{j=1}^{n}\left(d_{i j} / x_{j}^{\xi}-y_{i}^{\xi}\right)=0 \\
\Leftrightarrow \bigwedge_{j=1}^{n}\left(d_{i j}-y_{i}^{\xi} \cdot x_{j}^{\xi}\right) & =0 \Leftrightarrow \bigwedge_{j=1}^{n}\left[\mathbf{D}_{\mathbf{X Y}}-\left(\mathbf{y}^{\xi} \dot{\vee}\left(\mathbf{x}^{\xi}\right)^{\prime}\right)\right]_{i j}=0 .
\end{aligned}
$$

This last set of equations is true if and only if for each $\xi=1, \ldots, k$, and each integer $i=1, \ldots, \mathrm{m}$, each column entry of the $i_{t h}$ row of the matrix $\mathbf{D}_{\mathbf{X Y}}-\left[\mathbf{y}^{\xi} \dot{\vee}\left(\mathbf{x}^{\xi}\right)^{\prime}\right]$ contains at least one zero entry.

The two theorems show that sometimes $\mathbf{C}_{\mathbf{X Y}}$ or $\mathbf{D}_{\mathbf{X Y}}$ of $\mathrm{NR}^{2} \mathrm{FMAM}$ can realize perfect associative memories for $(\mathbf{X}, \mathbf{Y})$ when the condition in Theorem 1 or in Theorem 2 is satisfied. Therefore, we can utilize them in order to obtain much better effectiveness for hetero associative memories. 
Example 1. Assume that $\mathbb{U}=\mathbb{R}_{+}, \bigcirc=\cdot \ominus=/$, and

$$
\mathbf{X}=\left[\begin{array}{lll}
1 & 2 & 2 \\
4 & 2 & 2 \\
4 & 2 & 4
\end{array}\right], \quad \mathbf{Y}=\left[\begin{array}{lll}
1 & 2 & 1 \\
2 & 2 & 2 \\
1 & 1 & 1
\end{array}\right]
$$

According to the method of FMAM, the memories $\mathbf{A}_{\mathbf{X Y}}$ and $\mathbf{B}_{\mathbf{X Y}}$ are given by

$$
\mathbf{A}_{\mathbf{X Y}}=\mathbf{Y}_{\wedge}^{\prime} \mathbf{X}^{\prime}=\left[\begin{array}{lcr}
0.5 & 0.25 & 0.25 \\
1 & 0.5 & 0.5 \\
0.5 & 0.25 & 0.25
\end{array}\right], \mathbf{B}_{\mathbf{X Y}}=\mathbf{Y}_{\vee}^{\prime} \mathbf{X}^{\prime}=\left[\begin{array}{ccc}
1 & 1 & 1 \\
2 & 1 & 1 \\
1 & 0.5 & 0.5
\end{array}\right]
$$

Accordingly, their recall memories are

$$
\mathbf{A}_{\mathbf{X} \mathbf{Y}} \dot{\vee} \mathbf{X}=\left[\begin{array}{lll}
1 & 1 & 1 \\
2 & 2 & 2 \\
1 & 1 & 1
\end{array}\right] \neq \mathbf{Y}, \mathbf{B}_{\mathbf{X Y}} \wedge \mathbf{X}=\left[\begin{array}{lll}
1 & 2 & 2 \\
2 & 2 & 2 \\
1 & 1 & 1
\end{array}\right] \neq \mathbf{Y}
$$

Both $\mathbf{A}_{\mathbf{X Y}}$ and $\mathbf{B}_{\mathbf{X Y}}$ can not do the perfect recall memories for $(\mathbf{X}, \mathbf{Y})$.

According to the method of MAM,the memories $\mathbf{W}_{\mathbf{X Y}}$ and $\mathbf{M}_{\mathbf{X Y}}$ of MAM are given by

$$
\mathbf{W}_{\mathbf{X Y}}=\mathbf{Y}_{\wedge}^{-} \mathbf{X}^{\prime}=\left[\begin{array}{lll}
-1 & -3 & -3 \\
0 & -2 & -2 \\
-1 & -3 & -3
\end{array}\right], \mathbf{M}_{\mathbf{X Y}}=\mathbf{Y}^{-} \mathbf{X}^{\prime}=\left[\begin{array}{ccc}
0 & 0 & 0 \\
1 & 0 & 0 \\
0 & -1 & -1
\end{array}\right]
$$

Accordingly, their recall memories are

$$
\mathbf{W}_{\mathbf{X Y}} \stackrel{+}{\vee} \mathbf{X}=\left[\begin{array}{ccc}
1 & 1 & 1 \\
2 & 2 & 2 \\
1 & 1 & 1
\end{array}\right] \neq \mathbf{Y}, \mathbf{M}_{\mathbf{X Y}} \stackrel{+}{\wedge} \mathbf{X}=\left[\begin{array}{lll}
1 & 2 & 2 \\
2 & 2 & 2 \\
1 & 1 & 1
\end{array}\right] \neq \mathbf{Y} .
$$

Both $\mathbf{W}_{\mathbf{X Y}}$ and $\mathbf{M}_{\mathbf{X Y}}$ can't do the perfect recall memories for $(\mathbf{X}, \mathbf{Y})$.

But according to $\mathrm{NR}^{2} \mathrm{FMAM}$ method, the memories $\mathbf{C}_{\mathbf{X Y}}$ and $\mathbf{D}_{\mathbf{X Y}}$, as well as the according recall outputs are respectively given by

$$
\begin{aligned}
& \mathbf{C}_{\mathbf{X Y}}=\mathbf{Y} \dot{\wedge} \mathbf{X}^{\prime}=\left[\begin{array}{lll}
1 & 2 & 4 \\
2 & 4 & 4 \\
1 & 2 & 2
\end{array}\right], \mathbf{C}_{\mathbf{X Y}}{ }^{\prime} \mathbf{X}=\left[\begin{array}{lll}
1 & 2 & 1 \\
2 & 2 & 2 \\
1 & 1 & 1
\end{array}\right]=\mathbf{Y} . \\
& \mathbf{D}_{\mathbf{X Y}}=\mathbf{Y} \dot{\vee} \mathbf{X}^{\prime}=\left[\begin{array}{lll}
4 & 4 & 4 \\
4 & 8 & 8 \\
2 & 4 & 4
\end{array}\right], \mathbf{D}_{\mathbf{X Y}} \wedge \mathbf{X}=\left[\begin{array}{lll}
1 & 2 & 1 \\
2 & 2 & 2 \\
1 & 1 & 1
\end{array}\right]=\mathbf{Y} .
\end{aligned}
$$

The result shows that both $\mathbf{C}_{\mathbf{X Y}}$ and $\mathbf{D}_{\mathbf{X Y}}$ are perfect recall memories for $(\mathbf{X}, \mathbf{Y})$.

Our NR ${ }^{2}$ FMAM also has a certain ability to resist noise. $\mathbf{C}_{\mathbf{X Y}}$ resists dilative noise, and $\mathbf{D}_{\mathbf{X Y}}$ erosive noise. The following theorem shows this ability of $\mathrm{NR}^{2} \mathrm{FMAM}$, and simultaneously gives the boundary conditions of noise. 
Feng N., Yao Y.: No rounding reverse fuzzy morphological...

Theorem 3. Let $\tilde{\mathbf{x}}^{\gamma}$ denote the distorted version of the pattern $\mathbf{x}^{\gamma}, \gamma \in\{1, \ldots, k\}$. Then $\mathbf{C}_{\mathbf{X Y}} \vee^{\prime} \tilde{\mathbf{x}}^{\gamma}=\mathbf{y}^{\gamma}$, if and only if

$$
\tilde{x}_{j}^{\gamma} \geq x_{j}^{\gamma} \wedge \bigvee_{i=1}^{m}\left[\bigwedge_{\xi \neq \gamma}\left(y_{i}^{\xi} / y_{i}^{\gamma} \cdot x_{j}^{\xi}\right)\right] \forall j=1, \ldots, n
$$

and for each row index $i \in\{1, \ldots, m\}$ there exists a column index $j_{i} \in\{1, \ldots, n\}$ such that

$$
\tilde{x}_{j_{i}}^{\gamma}=x_{j_{i}}^{\gamma} \wedge\left[\bigwedge_{\xi \neq \gamma}\left(y_{i}^{\xi} / y_{i}^{\gamma} \cdot x_{j_{i}}^{\xi}\right)\right] \forall j=1, \ldots, n .
$$

Proof. 1) Suppose that $\tilde{\mathbf{x}}^{\gamma}$ denotes a distorted version of $\mathbf{x}^{\gamma}$ and that for $\gamma=$ $1, \ldots, k, \mathbf{C}_{\mathbf{X Y}} \vee^{\prime} \tilde{\mathbf{x}}^{\gamma}=\mathbf{y}^{\gamma}$. Then

$$
y_{i}^{\gamma}=\left(\mathbf{C}_{\mathbf{X Y}} \vee \tilde{\mathbf{x}}^{\gamma}\right)_{i}=\bigvee_{l=1}^{n}\left(c_{i l} / \tilde{x}_{l}^{\gamma}\right) \geq c_{i j} / \tilde{x}_{j}^{\gamma} \forall i=1, \ldots, m \text { and } \forall j=1, \ldots, n .
$$

Therefore

$$
\begin{aligned}
& \tilde{x}_{j}^{\gamma} \geq c_{i j} / y_{i}^{\gamma} \forall i=1, \ldots, m \text { and } \forall j=1, \ldots, n \\
\Leftrightarrow & \tilde{x}_{j}^{\gamma} \geq \bigvee_{i=1}^{m}\left(c_{i j} / y_{i}^{\gamma}\right) \quad \forall j=1, \ldots, n \\
\Leftrightarrow & \tilde{x}_{j}^{\gamma} \geq \bigvee_{i=1}^{m}\left[\bigwedge_{\xi=1}^{k}\left(y_{i}^{\xi} \cdot x_{j}^{\xi}\right) / y_{i}^{\gamma}\right] \quad \forall j=1, \ldots, n \\
\Leftrightarrow & \tilde{x}_{j}^{\gamma} \geq \bigvee_{i=1}^{m}\left[\bigwedge_{\xi \neq \gamma}\left(y_{i}^{\xi} \cdot x_{j}^{\xi} / y_{i}^{\gamma}\right) \wedge x_{j}^{\gamma}\right] \quad \forall j=1, \ldots, n \\
\Leftrightarrow & \tilde{x}_{j}^{\gamma} \geq x_{j}^{\gamma} \wedge \bigvee_{i=1}^{m}\left[\bigwedge_{\xi \neq \gamma}\left(y_{i}^{\xi} / y_{i}^{\gamma} \cdot x_{j}^{\xi}\right)\right] \quad \forall j=1, \ldots, n .
\end{aligned}
$$

This shows that the inequalities given by (25) are satisfied. It also follows that

$$
\tilde{x}_{j}^{\gamma} \geq x_{j}^{\gamma} \wedge\left[\bigwedge_{\xi \neq \gamma}\left(y_{i}^{\xi} / y_{i}^{\gamma} \cdot x_{j}^{\xi}\right)\right] \forall j=1, \ldots, n \text { and } \forall i=1, \ldots, m .
$$

Suppose that the set of inequalities given by (29) does not contain an equality for $i=1, \ldots, m$, namely, assume that there exists a row index $i \in\{1, \ldots, m\}$, such that

$$
\tilde{x}_{j}^{\gamma}>x_{j}^{\gamma} \wedge\left[\bigwedge_{\xi \neq \gamma}\left(y_{i}^{\xi} / y_{i}^{\gamma} \cdot x_{j}^{\xi}\right)\right] \forall j=1, \ldots, n .
$$


Then

$$
\begin{aligned}
& \left(\mathbf{C}_{\mathbf{X Y}} \dot{\vee}^{\prime} \tilde{\mathbf{x}}^{\gamma}\right)_{i}=\bigvee_{j=1}^{n}\left(c_{i j} / \tilde{x}_{j}^{\gamma}\right)<\bigvee_{j=1}^{n}\left[c_{i j} /\left(x_{j}^{\gamma} \wedge \bigwedge_{\xi \neq \gamma}\left(y_{i}^{\xi} \cdot x_{j}^{\xi} / y_{i}^{\gamma}\right)\right]=\right. \\
= & \bigvee_{j=1}^{n}\left[c_{i j} /\left(\bigwedge_{\xi=1}^{k}\left(y_{i}^{\xi} \cdot x_{j}^{\xi} / y_{i}^{\gamma}\right)\right)\right]=\bigvee_{j=1}^{n}\left[c_{i j} \cdot y_{i}^{\gamma} / \bigwedge_{\xi=1}^{k}\left(y_{i}^{\xi} \cdot x_{j}^{\xi}\right)\right]= \\
= & \bigvee_{j=1}^{n}\left[c_{i j} \cdot y_{i}^{\gamma} / c_{i j}\right]=y_{i}^{\gamma} .
\end{aligned}
$$

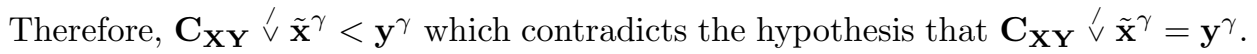
It follows that for each row index $i$ there must exist a column index $j_{i}$ satisfying (26).

2) Suppose that

$$
\tilde{x}_{j}^{\gamma} \geq x_{j}^{\gamma} \wedge \bigvee_{i=1}^{m}\left[\bigwedge_{\xi \neq \gamma}\left(y_{i}^{\xi} / y_{i}^{\gamma} \cdot x_{j}^{\xi}\right)\right] \forall j=1, \ldots, n
$$

By the first part of our proof, this inequality is true if and only if

$$
\tilde{x}_{j}^{\gamma} \geq c_{i j} / y_{i}^{\gamma} \forall i=1, \ldots, m \text { and } \forall j=1, \ldots, n
$$

or, equivalently, if and only if

$$
\begin{aligned}
& c_{i j} / \tilde{x}_{j}^{\gamma} \leq y_{i}^{\gamma} \quad \forall i=1, \ldots, m \text { and } \forall j=1, \ldots, n \\
& \Leftrightarrow \bigvee_{j=1}^{n}\left(c_{i j} / \tilde{x}_{j}^{\gamma}\right) \leq y_{i}^{\gamma} \forall i=1, \ldots, m
\end{aligned}
$$

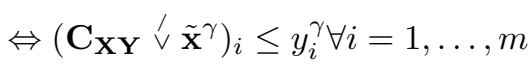

which implies that $\mathbf{C}_{\mathbf{X Y}} \grave{\vee}^{\prime} \tilde{\mathbf{x}}^{\gamma} \leq \mathbf{y}^{\gamma}, \forall \gamma=1, \ldots, k$. Thus, if we can show that $\mathbf{C}_{\mathbf{X Y}} \dot{\vee}^{\prime} \tilde{\mathbf{x}}^{\gamma} \geq \mathbf{y}^{\gamma}, \forall \gamma=1, \ldots, k$, then we must improve that $\mathbf{C}_{\mathbf{X Y}} \stackrel{\vee}{\vee}^{\gamma} \tilde{\mathbf{x}}^{\gamma}=\mathbf{y}^{\gamma}, \forall \gamma=$ $1, \ldots, k$. Let $\gamma \in\{1, \ldots, k\}$ and $i \in\{1, \ldots, m\}$ be arbitrarily chosen. Then

$$
\begin{aligned}
\left(\mathbf{C}_{\mathbf{X Y}} \vee \tilde{\mathbf{x}}^{\gamma}\right)_{i} & =\bigvee_{j=1}^{n}\left(c_{i j} / \tilde{x}_{j}^{\gamma}\right) \geq c_{i j_{i}} / \tilde{x}_{j_{i}}^{\gamma}=c_{i j_{i}} /\left[x_{j_{i}}^{\gamma} \wedge \bigwedge_{\xi \neq \gamma}\left(y_{i}^{\xi} / y_{i}^{\gamma} \cdot x_{j_{i}}^{\xi}\right)\right] \\
& \left.=c_{i j_{i}} /\left[\bigwedge_{\xi=1}^{k}\left(y_{i}^{\xi} / y_{i}^{\gamma} \cdot x_{j_{i}}^{\xi}\right)\right]=c_{i j_{i}} \cdot y_{i}^{\gamma} / \bigwedge_{\xi=1}^{k}\left(y_{i}^{\xi} \cdot x_{j_{i}}^{\xi}\right)\right] \\
& =c_{i j_{i}} \cdot y_{i}^{\gamma} / c_{i j_{i}}=y_{i}^{\gamma} .
\end{aligned}
$$

This shows that $\mathbf{C}_{\mathbf{X Y}} \mathfrak{\vee}^{\mathbf{\mathbf { x }}} \tilde{\mathbf{y}}^{\gamma}$. 
Theorem 4. Let $\tilde{\mathbf{x}}^{\gamma}$ denote the distorted version of the pattern $\mathbf{x}^{\gamma}$. Then $\mathbf{D}_{\mathbf{X Y}} \wedge$ $\tilde{\mathbf{x}}^{\gamma}=\mathbf{y}^{\gamma}$, if and only if

$$
\tilde{x}_{j}^{\gamma} \leq x_{j}^{\gamma} \vee \bigwedge_{i=1}^{m}\left[\bigvee_{\xi \neq \gamma}\left(y_{i}^{\xi} / y_{i}^{\gamma} \cdot x_{j}^{\xi}\right)\right] \forall j=1, \ldots, n
$$

and for each row index $i \in\{1, \ldots, m\}$ there exists a column index $j_{i} \in\{1, \ldots, n\}$ such that

$$
\tilde{x}_{j_{i}}^{\gamma}=x_{j_{i}}^{\gamma} \vee\left[\bigvee_{\xi \neq \gamma}\left(y_{i}^{\xi} / y_{i}^{\gamma} \cdot x_{j_{i}}^{\xi}\right)\right] .
$$

Proof. Because the proof of Theorem 4 is similar to the proof of Theorem 3, so it is omitted here.

Example 2. Let

$$
\begin{aligned}
& \mathbf{X}=\left[\begin{array}{lll}
1 & 2 & 2 \\
4 & 2 & 2 \\
4 & 2 & 4
\end{array}\right], \mathbf{Y}=\left[\begin{array}{lll}
2 & 4 & 2 \\
4 & 4 & 4 \\
2 & 2 & 2
\end{array}\right], \overline{\mathbf{X}}=\left[\begin{array}{lll}
1 & 2 & 1 \\
2 & 2 & 2 \\
4 & 2 & 4
\end{array}\right], \\
& \widetilde{\mathbf{X}}=\left[\begin{array}{lcr}
0.1 & 2 & 1 \\
0.001 & 0.001 & 0.002 \\
4 & 0.002 & 4
\end{array}\right], \widehat{\mathbf{X}}=\left[\begin{array}{lcr}
100 & 200 & 100 \\
2 & 2000 & 2 \\
4000 & 2 & 40
\end{array}\right],
\end{aligned}
$$

where $\overline{\mathbf{X}}, \widetilde{\mathbf{X}}$ and $\widehat{\mathbf{X}}$ denote a distorted version of $\mathbf{X}$, respectively. The recall results of $\mathbf{W}_{\mathbf{X Y}}$ and $\mathbf{M}_{\mathbf{X Y}}$ of MAM, $\mathbf{A}_{\mathbf{X Y}}$ and $\mathbf{B}_{\mathbf{X Y}}$ of FMAM, and $\mathbf{C}_{\mathbf{X Y}}$ and $\mathbf{D}_{\mathbf{X Y}}$ of $\mathrm{NR}^{2}$ FMAM in the Example 2 are given in the Tab. I. From the table we can see that the recall result of MAM is the worst. Its correct recall rate in the Example 2 is zero. The best recall result belongs to $\mathrm{NR}^{2} \mathrm{FMAM}$. At the same time, the $\mathbf{C}_{\mathbf{X Y}}$ of $\mathrm{NR}^{2} \mathrm{FMAM}$ has a good ability to deal with dilative noise when the dilative input is presented to $\mathrm{NR}^{2} \mathrm{FMAM}$; and the $\mathbf{D}_{\mathbf{X Y}}$ has a good ability to deal with erosive noise when the erosive input is presented to $\mathrm{NR}^{2} \mathrm{FMAM}$.

\begin{tabular}{lcccc}
\hline Input & $\mathbf{X}$ & $\overline{\mathbf{X}}$ & $\widetilde{\mathbf{X}}$ & $\widehat{\mathbf{X}}$ \\
\hline Recall of $\mathbf{W}_{\mathbf{X Y}}$ & $\neq \mathbf{Y}$ & $\neq \mathbf{Y}$ & $\neq \mathbf{Y}$ & $\neq \mathbf{Y}$ \\
Recall of $\mathbf{M}_{\mathbf{X Y}}$ & $\neq \mathbf{Y}$ & $\neq \mathbf{Y}$ & $\neq \mathbf{Y}$ & $\neq \mathbf{Y}$ \\
Recall of $\mathbf{A}_{\mathbf{X Y}}$ & $\neq \mathbf{Y}$ & $\neq \mathbf{Y}$ & $\neq \mathbf{Y}$ & $\neq \mathbf{Y}$ \\
Recall of $\mathbf{B}_{\mathbf{X Y}}$ & $\neq \mathbf{Y}$ & $=\mathbf{Y}$ & $\neq \mathbf{Y}$ & $\neq \mathbf{Y}$ \\
Recall of $\mathbf{C}_{\mathbf{X Y}}$ & $=\mathbf{Y}$ & $=\mathbf{Y}$ & $\neq \mathbf{Y}$ & $=\mathbf{Y}$ \\
Recall of $\mathbf{D}_{\mathbf{X Y}}$ & $=\mathbf{Y}$ & $=\mathbf{Y}$ & $=\mathbf{Y}$ & $\neq \mathbf{Y}$ \\
\hline
\end{tabular}

Tab. I The recall results of MAM, FMAM and NR FMAM in the Example 2. 
Example 3. In the experiment, $\mathbf{X}=\{\mathrm{B}, \mathrm{C}, \mathrm{F}, \mathrm{H}, \mathrm{S}, \mathrm{W}\}, \mathbf{Y}=\{\mathrm{AI}$, Child, Dog, Flower, Illusion, Sheep $\}$. Each character in $\mathbf{X}$ is a $35 \times 35$ Boolean image, and each image in $\mathbf{Y}$ is a $50 \times 50$ gray image (as shown in Fig. 1, where the top row is the input patterns and the bottom row the target patterns). Because of the domain $\mathbb{U}>0$ for FMAM and NR ${ }^{2}$ FMAM, in order to avoid zero value elements, we can make an appropriate processing, such as each zero element is set to a small positive number.

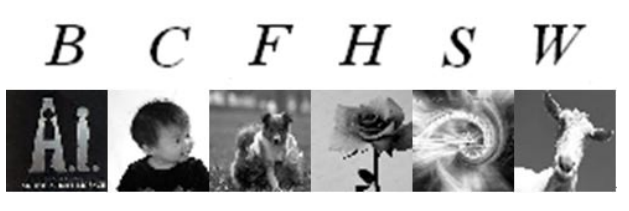

Fig. 1 Original associative memory patterns.

Experiment shows that for FMAM, both $\mathbf{A}_{\mathbf{X Y}}$ and $\mathbf{B}_{\mathbf{X Y}}$ cannot perfectly recall memories with the worst outcome, i.e. zero recall rate (see Fig. 2, where the top row shows the recall patterns of the $\mathbf{A}_{\mathbf{X Y}}$ and the bottom row the recall patterns of the $\mathbf{B}_{\mathbf{X Y}}$ ). The result comes from strict procedure analysis, rather than only by visual inspection. The analysis standard is that a recall image is incomplete as long as a pixel is different from the pixel of original image.

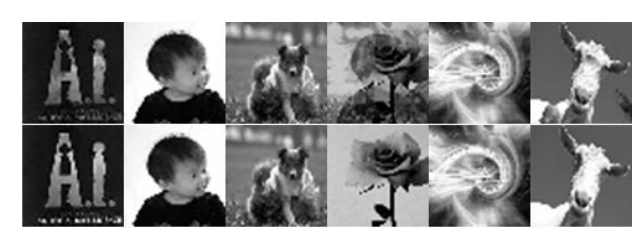

Fig. 2 Experimental result of FMAM

The experimental result of MAM (or RMAM) is shown in Fig. 3, where the top row shows the recall patterns of the $\mathbf{W}_{\mathbf{X Y}}$ and the bottom row the recall patterns of the $\mathbf{M}_{\mathbf{X Y}}$. It shows that the correct recall rate of $\mathbf{W}_{\mathbf{X Y}}$ was $17 \%$ (only Child can be completely recalled), and the correct recall rate of $\mathbf{M}_{\mathbf{X Y}}$ was $83 \%$ (only the AI cannot be completely recalled).

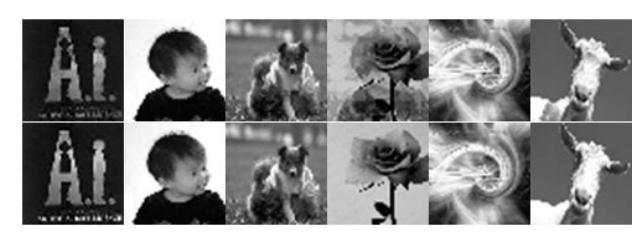

Fig. 3 Experimental result of MAM.

The experimental result of $\mathrm{NR}^{2} \mathrm{FMAM}$ is shown in Fig. 4: the top row is the recall patterns of the $\mathbf{C}_{\mathbf{X Y}}$ and the bottom row the recall patterns of the $\mathbf{D}_{\mathbf{X Y}}$. It shows that the correct recall rate of $\mathbf{D}_{\mathbf{X Y}}$ was $17 \%$ (only Child can be completely recalled). 
Feng N., Yao Y.: No rounding reverse fuzzy morphological...

But amazingly, $\mathbf{C}_{\mathbf{X Y}}$ can achieve perfect recall memories with correct rate of $100 \%$ ! Experimental result is encouraging! Our experiments demonstrate that in quite a number of cases $\mathrm{NR}^{2} \mathrm{FMAM}$ has better effect than FMAM and MAM on hetero associative memories. Therefore, the method is a good complement to FMAM and MAM.

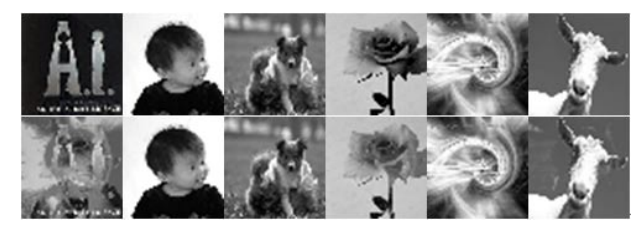

Fig. 4 Experimental result of $N R^{2} F M A M$.

\section{Discussions}

Why can $\mathrm{NR}^{2} \mathrm{FMAM}$ in many cases get better results than FMAM? What is its application in the social network? We discussed these questions from the following three aspects.

\subsection{No rounding}

We think that the different operation order and no rounding in memory and recall may be one of possible reasons. In theory, $a \times b / b=a$, and $a / b \times b=a$, i.e. $a \times b / b=$ $a / b \times b$, but in practice and for computers, it may not necessarily turn out that way. In the case of no overflow and operating from left to right, $a \times b / b=a$, which operation result is no problem. This operation is from multiplication to division (FMTD) like in NR $\mathrm{NRMAM}^{2}$. But that computing result of $a / b \times b$ is uncertain. We know that computing accuracy of a computer is limited by its word length. In the case of indivisible, the operation of the computer may bring us the error, such that $a / b \times b \neq a$ or $a / b \times b \approx a$ because of the round off. This operation is from division to multiplication (FDTM) with round off. The operation in FMAM just is of FDTM. Therefore, for hetero associative morphological memories, we more inclined to adopt $\mathrm{NR}^{2} \mathrm{FMAM}$ method. Of course, this method does not preclude FMAM method. In general, they should be complementary.

\subsection{The conditions which $\mathrm{NR}^{2}$ FMAM is better than FMAM}

Let $\left(\mathbf{x}^{1}, \mathbf{y}^{1}\right), \ldots,\left(\mathbf{x}^{k}, \mathbf{y}^{k}\right)$ be $k$ pairs of vector patterns for associative memories, $\mathbf{X}=\left(\mathbf{x}^{1}, \ldots, \mathbf{x}^{k}\right)$ the input pattern matrix, and $\mathbf{Y}=\left(\mathbf{y}^{1}, \ldots, \mathbf{y}^{k}\right)$ the output pattern matrix. Assume that $R_{R}$ is the correct recall rate of $\mathrm{NR}^{2} \mathrm{FMAM}$, and $R_{F}$ the correct recall rate of FMAM. Then, what are the conditions of that $R_{R}>R_{F}$ ? The next theorem answers this question. 
Theorem 5. $R_{R}>R_{F}$ if and only if the following conditions are satisfied:

1) In $k$ pairs of vector patterns, there exist $m$ pairs of vector patterns which satisfy that for each $\xi_{i} \in\{1, \ldots, k\}$ and $i=1, \ldots, m$, each row of the matrix $\left[\mathbf{y}^{\xi_{i}}\right.$ $\left.\dot{\wedge}\left(\mathbf{x}^{\xi_{i}}\right)^{\prime}\right]-\mathbf{C}_{\mathbf{X Y}}$ contains a zero entry, or each row of the matrix $\mathbf{D}_{\mathbf{X Y}}-\left[\mathbf{y}^{\xi_{i}}\right.$ $\left.\dot{v}\left(\mathbf{x}^{\xi_{i}}\right)^{\prime}\right]$ contains a zero entry.

2) In $k$ pairs of vector patterns, there exist $n$ pairs of vector patterns which satisfy that for each $\xi_{j} \in\{1, \ldots, k\}$ and $j=1, \ldots, n$, each row of the matrix $\left[\mathbf{y}^{\xi_{j}}\right.$ $\left.\wedge^{\prime}\left(\mathbf{x}^{\xi_{j}}\right)^{\prime}\right]-\mathbf{A}_{\mathbf{X Y}}$ contains a zero entry, or each row of the matrix $\mathbf{B}_{\mathbf{X Y}}-\left[\mathbf{y}^{\xi_{j}}\right.$ $\left.\hat{\vee}\left(\mathbf{x}^{\xi_{j}}\right)^{\prime}\right]$ contains a zero entry.

3) $m>n$.

Proof. 1) Assume that $m>n$. According to Theorem 1, we know that $m$ pairs of vectors in $k$ pairs of vectors satisfy that for each $\xi_{i} \in\{1, \ldots, k\}$ and $i=1, \ldots, m$, each row of the matrix $\mathbf{D}_{\mathbf{X Y}}-\left[\mathbf{y}^{\xi_{i}} \dot{\vee}\left(\mathbf{x}^{\xi_{i}}\right)^{\prime}\right]$ contains a zero entry or each row of the matrix $\left[\mathbf{y}^{\xi_{i}} \dot{\wedge}\left(\mathbf{x}^{\xi_{i}}\right)^{\prime}\right]-\mathbf{C}_{\mathbf{X Y}}$ contains a zero entry means that $m$ pairs of vectors can be correctly associated and recalled, and the recall rate $R_{R}=m / k$; in addition, according to FMAM, we know that $n$ pairs of vectors in $k$ pairs of vectors satisfy that for each $\xi_{j} \in\{1, \ldots, k\}$ and $j=1, \ldots, n$, each row of the matrix $\left[\mathbf{y}^{\xi_{j}} \wedge\left(\mathbf{x}^{\xi_{j}}\right)^{\prime}\right]-\mathbf{A}_{\mathbf{X Y}}$ contains a zero entry or each row of the matrix $\mathbf{B}_{\mathbf{X Y}}-\left[\mathbf{y}^{\xi_{j}} \vee\left(\mathbf{x}^{\xi_{j}}\right)^{\prime}\right]$ contains a zero entry means that $n$ pairs of vectors can be correctly associated and recalled, and the recall rate $R_{F}=n / k$. Since $m>n$, we have that $R_{R}>R_{F}$.

2) Assume that $R_{R}>R_{F}$, might as well set $R_{R}=m / k, R_{F}=n / k$, and $m>$ $n$. It means that $m$ pairs of vectors in $k$ pairs of vectors can be correctly associated and recalled by $\mathrm{NR}^{2} \mathrm{FMAM}$ and $n$ pairs of vectors in $k$ pairs of vectors can be correctly associated and recalled by FMAM. According to the Theorem 1 we know that $m$ pairs of vectors satisfy that for each $\xi_{i} \in$ $\{1, \ldots, k\}$ and $i=1, \ldots, m$, each row of the matrix $\left[\mathbf{y}^{\xi_{i}} \dot{\wedge}\left(\mathbf{x}^{\xi_{i}}\right)^{\prime}\right]-\mathbf{C}_{\mathbf{X Y}}$ contains a zero entry or each row of the matrix $\mathbf{D}_{\mathbf{X Y}}-\left[\mathbf{y}^{\xi_{i}} \dot{\vee}\left(\mathbf{x}^{\xi_{i}}\right)^{\prime}\right]$ contains a zero entry. In addition, according to FMAM, it is certain that $n$ pairs of vectors satisfy that for each $\xi_{j} \in\{1, \ldots, k\}$ and $j=1, \ldots, n$, each row of the matrix $\left[\mathbf{y}^{\xi_{j}} \wedge\left(\mathbf{x}^{\xi_{j}}\right)^{\prime}\right]-\mathbf{A}_{\mathbf{X Y}}$ contains a zero entry or each row of the matrix $\mathbf{B}_{\mathbf{X Y}}-\left[\mathbf{y}^{\xi_{j}} \vee\left(\mathbf{x}^{\xi_{j}}\right)^{\prime}\right]$ contains a zero entry.

\subsection{Its application in the social network}

We should point out that $\mathrm{NR}^{2} \mathrm{FMAM}$ has these functions of pattern recognition, clustering, classification and prediction. If $\mathbf{Y}=\mathbf{X}, \mathrm{NR}^{2} \mathrm{FMAM}$ is the auto association from $\mathbf{X}$ to $\mathbf{X}$. Then take advantage of its anti-noise function, we can 
Feng N., Yao Y.: No rounding reverse fuzzy morphological...

realize pattern recognition and clustering analysis. Next, we can realize the classification and prediction by using the hetero associative from $\mathbf{X}$ to $\mathbf{Y}$. In a social network $[15,18,26]$, the identification of a social group and the group leader's recognition are an important research content, and this recognition depends on the clustering and classification techniques. In the present, improving the identification effect of community organizations and community leaders is still needed [27, 28]. Obviously, NR ${ }^{2}$ FMAM can serve as a new identification technology in social networks, and we should study and use it deeply.

\section{Conclusion}

In this paper, aiming at the shortcomings of hetero associative morphological memories, we propose the $\mathrm{NR}^{2} \mathrm{FMAM}$ method. Theoretical analysis shows that $\mathrm{NR}^{2} \mathrm{FMAM}$ under certain conditions can realize perfect recall memories, at the same time with a certain noise robustness. Experiments have demonstrated that, in many cases, $\mathrm{NR}^{2} \mathrm{FMAM}$ has better effects of hetero associative morphological memories than FMAM. Why is this? The paper has some discussions for this problem. No rounding is one of those possible reasons that result in higher recall rate than FMAM. The conditions of $R_{R}>R_{F}$, namely the conditions of $\mathrm{NR}^{2} \mathrm{FMAM}$ is better than FMAM are given by Theorem 5 . For hetero associative morphological memories, we tend to use NR ${ }^{2}$ FMAM. Of course, it should be pointed out that $\mathrm{NR}^{2}$ FMAM and FMAM are complementary, not exclusive. Anyway, $\mathrm{NR}^{2}$ FMAM is a beneficial way for HAMM. We believe that combining NR ${ }^{2}$ FMAM with FMAM can significantly improve the effectiveness of HAMM, and solve more problems in practical applications. In the social network, we can find the application of NR ${ }^{2}$ FMAM. Of course, this problem needs to be further studied in the future.

\section{Acknowledgement}

This work was supported in part by the National Science Foundations of China (61173071), in part by the science and technology research project of Zhengzhou city (153PKJGG153).

\section{References}

[1] CHEN S.C., LIU W.L. Complex morphological associative memories and their performance analysis. Journal of Software. 2002, 13(3), pp. 453-459, doi: 10.13328/j.cnki.jos.2002. 03.019

[2] FENG N.Q., AO L.H., WANG S.X., WANG S.X., TIAN Y. Application of morphological associative memories to the associative recognition for images. Journal of Henan Normal University (Natural Science) . 2010, 38(3), pp. 44-47 (in Chinese with English abstract), doi: $10.16366 / j . c n k i .1000-2367.2010 .03 .052$.

[3] FENG N.Q., LIU C.H., ZHANG C.P., XU J.C., WANG S.X. Research on the framework of morphological associative memories. Chinese Journal of Computers. 2010, 33(1), pp. 157166, doi: $10.3724 /$ SP. J.1016.2010.00157.

[4] FENG N.Q., QIN L.J., WANG X.F., TIAN Y., ZHU X. Morphological associative memories applied to the implicit learning. Journal of Henan Normal University (Natural Science) . 2013, 41(3), pp. 156-159 (in Chinese with English abstract), doi: 10.16366/j.cnki. 1000-2367.2013.03.030. 


\section{Neural Network World 6/2016, 571-587}

[5] FENG N.Q., TIAN Y., WANG X.F., QIN L.J., QIAO K. Grouping of morphological heteroassociative memories. Journal of Henan Normal University (Natural Science) . 2012, 40(2), pp.155-158 (in Chinese with English abstract), doi: 10.16366/j.cnki.1000-2367.2012.02. 003.

[6] FENG N.Q, TIAN Y., WANG X.F., SONG L.M., FAN H.J., WANG S.X. Logarithmic and exponential morphological associative memories. Ruan Jian Xue Bao/Journal of Software. 2015, 26(7), pp. 1662-1674 (in Chinese with English abstract). http://www.jos.org.cn/ 1000-9825/4620.htm, doi: 10.13328/j.cnki.jos.004620.

[7] FENG N.Q., WANG X.F., MAO W.T., AO L. Heteroassociative Morphological Memories Based on Four-Dimensional Storage. Neurocomputing. 2013, 116, pp. 76-86, doi: 10.1016/ j.neucom.2012.01.043.

[8] HAGAN M.T., DEMUTH H.B., BEALE M.H. Neural network design. Beijing: China Machine Press, CITIC PUBLISHING HOUSE, 2002.

[9] Hopfield J.J. Neural networks and physical systems with emergent collective computational abilities. In: Proc. Nat. Acad. Sci.. 1982, 79(8), pp. 2554-2558, doi: 10.1073/pnas.79.8. 2554.

[10] HUANG D.S. Radial basis probabilistic neural networks: Model and application. Int. Journal of Pattern Recognition and Artificial Intelligence. 1999, 13(7), pp. 1083-1101, doi: 10.1142/ S0218001499000604.

[11] HUANG D.S. Systematic Theory of Neural Networks for Pattern Recognition (in Chinese). Beijing: Publishing House of Electronic Industry of China. May 1996.

[12] HUANG D.S., DU J.X. A constructive hybrid structure optimization methodology for radial basis probabilistic neural networks. IEEE Trans. Neural Networks. 2008, 19(12), pp. 20992115, doi: 10.1109/TNN. 2008.2004370.

[13] HUANG D.S., JIANG W. A general CPL-AdS methodology for fixing dynamic parameters in dual environments. IEEE Trans. on Systems, Man and Cybernetics - Part B. 2012, 42(5), pp. 1489-1500, doi: 10.1109/TSMCB.2012.2192475.

[14] HUANG D.S., ZHENG H.H. Independent component analysis based penalized discriminant method for tumor classification using gene expression data. Bioinformatics. 2006, 22(15), 4, pp. 1855-1862, doi: 10.1093/bioinformatics/btl190.

[15] NEWMAN M.E., GIRVAN M. Finding and evaluating community structure in networks. Physical Review E. 2004, 69(2), pp. 026113, doi: 10.1103/PhysRevE.69.026113.

[16] RITTER G.X., DIAZ-DE-LEON J.L., SUSSNER P. Morphological bidirectional associative memories. Neural Networks. 1999, 12(6), pp. 851-867, doi: 10.1016/S0893-6080 (99) 00033-7

[17] RITTER G.X., SUSSNER P., DIAZ-DE-LEON J.L. Morphological Associative Memories. IEEE Transactions on Neural Networks. 1998, 9(2), pp. 281-293, doi: 10.1109/72.661123.

[18] SCOTT J. Social Network Analysis: A Handbook. Sage Publications, London, 2000.

[19] SUSSNER P., VALLE M.E. Gray-scale morphological associative memories. IEEE Transactions on Neural Networks. 2006, 17(3), pp. 559-570, doi: 10.1109/TNN.2006.873280.

[20] SUSSNER P., VALLE M.E. Morphological and certain fuzzy morphological associative memories with applications in classification and prediction. Computational Intelligence Based on Lattice Theory, Series: Studies in Computational Intelligence. 2007, vol. 67, pp. 149-171, doi: 10.1007/978-3-540-72687-6_8.

[21] VÁZQUEZ R.A., SOSSA H. Morphological Hetero-Associative Memories Applied to Restore True-Color Patterns. Lecture Notes In Computer Science. 2009, vol. 5553, pp. 520-529, doi: 10.1007/978-3-642-01513-7_57.

[22] WANG M., CHEN S.C. Enhanced FMAM based on empirical kernel map. IEEE Transactions on Neural Networks. 2005, 16(3), pp. 557-564, doi: 10.1109/TNN.2005.847839.

[23] WANG M., CHU R. Economizing Enhanced Fuzzy Morphological Associative Memory. In: International Conference on Machine Learning and Cybernetics. 2008, Vol. 1, pp. 495-500, doi: 10.1109/ICMLC. 2008.4620455. 
Feng N., Yao Y.: No rounding reverse fuzzy morphological. . .

[24] WANG M., WANG S.T., WU X.J. Initial results on fuzzy morphological associative memories. Acta Electronica Sinica. 2003, 31(5), pp. 690-693. http://www.ejournal.org.cn/CN/ abstract/abstract2167. shtml

[25] WANG X.F., HUANG D.S., XU H. An efficient local Chan-Vese model for image segmentation. Pattern Recognition. 2010, 43(3), pp. 603-618, doi: 10.1016/j.patcog.2009.08.002.

[26] WU F., HUBERMAN B.A. Finding communities in linear time: A physics approach. Phys. J. B. 2003, 38, pp. 331-338, doi: 10.1140/epjb/e2004-00125-x.

[27] ZHANG Z.Y., LIAN S.G., PEI Q.Q., et a1. Fuzzy Risk Assessments on Security Policies for Digital Rights Management. Neural Network World. 2010, 20(3), pp. 265-284. https: //www.highbeam.com/doc/1P3-2215800731.html

[28] ZHANG Z.Y., WANG K.L. A Trust Model for Multimedia Social Networks. Social Networks Analysis and Mining (Springer). 2013, 3, pp. 969-979, doi: 10.1007/s13278-012-0078-4. 
